\title{
Análise da relação entre sintomas urinários e topografia da lesão cerebral em pacientes com acidente vascular cerebral
}

\author{
Analysis of the relationship between urinary symptoms and the topography of brain injury \\ in stroke patients
}

\author{
Viliane Lourdes Banaszeskia, Paulo Pereira Christo ${ }^{b}$ \\ a Enfermeira. Mestre em Ciências da Saúde - Biomedicina. Enfermeira da Rede SARAH de Hospitais de Reabilitação, Belo Horizonte, Brasil. \\ b Médico. Doutor em neurologia pela Universidade de São Paulo. Professor da pós-graduação da Santa Casa de Belo Horizonte.
}

RESUMO

\begin{abstract}
Objetivo: Analisar a relação entre a topografia da lesão cerebral e disfunção vesical em pacientes com acidente vascular cerebral (AVC) isquêmico e hemorrágico comparando a região cerebral afetada com os sintomas do trato urinário inferior (STUI).
\end{abstract}

Materiais e Métodos: Estudo retrospectivo, desenvolvido no Hospital da Rede SARAH de Neurorreabilitação, Brasil, com 132 pacientes com AVC crônico atendidos no programa de reabilitação neurológica adulto, no período de janeiro de 2008 a dezembro de 2011.

Resultados: Cento e seis pacientes (80,3\%) apresentaram sequela de acidente vascular cerebral isquêmico e 26 $(19,7 \%)$ acidente vascular cerebral hemorrágico. A média de idade foi de 56 $\pm 13,1$ anos e o tempo médio da ocorrência do AVC foi de 21,4 meses. Em relação ao comportamento miccional, 35,6\% $(n=47)$ eram continentes vesicais, $45,5 \%(n=60)$ com sintomas do trato urinário inferior, e 18,9\% $(n=25)$ com incontinência urinária contínua. Observou-se que na ocorrência de lesões extensas acometendo a área frontal-parietal-temporal-occiptal $(p=0,001)$, alterações cognitivas $(p=0,001)$ e afasia $(p=0,001)$ houve relação estatística significativa com a presença de incontinência urinária contínua nestes pacientes. Nos pacientes com lesão fronto-parietal houve associação com o sintoma urgência $(p=0,050)$, urgeincontinência urinária $(p=0,042)$, incontinência urinária de esforço $(p=0,019)$ e polaciúria $(p=0,042)$.

Conclusão: Segundo a amostra estudada, observamos que alterações cognitivas e afasia tiveram correlação com a incontinência urinária contínua. Em relação a topografia de lesão cerebral houve associação entre lesões em região fronto-parietal com os sintomas de urgência, urgeincontinência, incontinência de esforço e polaciúria; e na ocorrência de lesão cerebral extensa houve prevalência de incontinência urinária contínua.

Palavras-chave: acidente vascular cerebral; transtornos urinários; lesões cerebrais.

Objective: This study analyzed the relation between brain injury topography and bladder dysfunction in patients with ischemic and hemorrhagic stroke by comparing the brain region affected with lower urinary tract symptoms (LUTS).

Material and Methods: A retrospective study developed by the SARAH Network of Rehabilitation Hospitals in Brazil, involving 132 patients with chronic stroke attended by an adult neurological rehabilitation program, from January 2008 to December 2011.

Results: 106 patients (80.3\%) showed ischemic stroke sequelae and 26 (19.7\%) hemorrhagic stroke sequelae. Patient mean age was $56.0 \pm 13.1$ years and the mean period since stroke occurrence was 21.4 months. Regarding voiding behavior, $35.6 \%$ ( $n=47)$ showed bladder continence, $45.5 \%(n=60)$ presented LUTS, and 18.9\% $(n=25)$ presented continuous urinary incontinence. The presence of continuous urinary incontinence was significantly associated with extensive lesions in the frontal-parietal-temporal-occipital regions $(p=0.001)$, cognitive deficits $(p=0.001)$ and aphasia $(p=0.001)$. In patients with frontoparietal lesions, associations were observed for urgency $(p=0.05)$, urge urinary incontinence $(p=0.042)$, urinary incontinence $(p=0.019)$ and pollakisuria $(p=0.042)$.

Conclusion: Data showed that cognitive impairment and aphasia were correlated with continuous urinary incontinence. Regarding brain injury topography, associations were determined between lesions to the frontal-parietal region and symptoms of urgency, urge urinary incontinence, stress urinary incontinence and pollakisuria, while extensive brain injury was associated with continuous urinary incontinence.

Keywords: stroke; urination disorders; brain injuries.

\section{Correspondência:}

VILIANE LOURDES BANASZESKI

Rua Ulisses Marcondes Escobar, 15, ap. 401 - Bairro Buritis

30575-110 Belo Horizonte, MG, Brasil

E-mail: vylyane@gmail.com 


\section{INTRODUÇÃO}

O acidente vascular cerebral (AVC) é uma das principais causas de incapacidade neurológica em adultos ${ }^{1}$ e danos cerebrais ocasionados por esse tipo de lesão podem resultar em alterações no comportamento vesical, interferindo na qualidade de vida desses indivíduos². Disfunção miccional pode estar presente em indivíduos com AVC isquêmico ou hemorrágico, podendo ser temporária ou permanente, dependendo do tamanho e da área de lesão. Sintomas urinários podem estar relacionados com afasias, déficit cognitivo, comportamental e motor ${ }^{3,4}$, os quais são relatados pelos profissionais de saúde, familiares e cuidadores para a avaliação da equipe de saúde se esta condição trata-se de disfunção do trato urinário inferior ${ }^{5}$.

Através da utilização de métodos de imagem como tomografia computadorizada por emissão de fóton único (SPECT) para mapear as regiões que são ativadas durante o processo da micção, foram observadas atividades significativas no giro frontal inferior direito e giro do cíngulo anterior direito durante a fase da micção ${ }^{6}$.

Alterações urinárias são comumente associadas com o acidente vascular cerebral, as quais diferem na fase aguda e crônica da lesão cerebral e também entre o tipo isquêmico e hemorrágico ${ }^{7}$. A incontinência e retenção urinária ocorrem com maior frequência nos primeiros três meses após o AVC. Os sintomas mais frequentes são noctúria, urgeincontinência e hesitação ${ }^{6}$. A incontinência afeta o bem-estar físico e social dos pacientes de forma negativa, influenciando na autoestima, maior demanda nos cuidados e ainda predispõe ao risco de complicações como infecções do trato urinário (ITU) inferior e alto ${ }^{8,9}$. A maioria dos estudos referem-se ao sintoma de incontinência urinária, no entanto, as demais queixas como a frequência, urgência, noctúria, disúria e esforço miccional tem um considerável impacto na vida dos pacientes, com igual ou maior reflexo em sua qualidade de vida ${ }^{4}$.

Alterações miccionais podem interferir na qualidade de vida, principalmente da população idosa institucionalizada. No Brasil a prevalência de incontinência urinária descrita na literatura varia de $2,5 \%$ a $60 \%$, as causas são várias, desde doenças crônicas, envelhecimento e número de gestações ${ }^{10}$. Os gastos com tratamentos médicos e demais cuidados são custeados pelos pacientes (absorventes, fraldas e outros dispositivos) e sistema público de saúde. Estima-se que ocorram cerca de um milhão de novos casos de bexiga hiperativa por ano, consequentemente os custos com avaliação diagnóstica e tratamento aumentam sendo considerado um problema de saúde pública. A implementação de ações a nível primário e secundários devem ser planejadas, visando a melhoria na qualidade de vida destes indivíduos e acima de tudo redução com gastos a nível terciário ${ }^{10,11,12}$.

O objetivo deste estudo foi analisar a relação entre a topografia da lesão cerebral em pacientes com AVC isquêmico e hemorrágico, com os sintomas do trato urinário inferior, apresentados pelo paciente.

\section{MATERIAIS E MÉTODOS}

Um estudo epidemiológico retrospectivo e analítico, foi desenvolvido por meio da avaliação dos dados em prontuário eletrônico dos pacientes com diagnóstico de acidente vascular cerebral isquêmico e hemorrágico, atendidos na Rede SARAH de Hospitais de Reabilitação em Belo Horizonte/MG, Brasil, no período de janeiro de 2008 a dezembro de 2011. O estudo foi aprovado pelo Comitê de Ética em Pesquisa, CAAE: 16794313.0.0000.5138.

Para a seleção destes pacientes foi utilizado os códigos de I-60 ao I-69 da Classificação Internacional de Doenças $(\mathrm{CID} 10)^{13}$, que tratam das doenças cerebrovasculares. Obtivemos 1.300 pacientes dos quais foram analisados 337 prontuários de forma aleatória e sequencial. Destes, 132 pacientes foram selecionados para participarem do estudo após preencher os critérios de inclusão e 205 foram excluídos.

Como critério de inclusão os pacientes deveriam ter 06 meses ou mais de lesão cerebral ${ }^{14}$, o qual foi calculado entre a data de admissão no Programa de Reabilitação Neurológica Adulto (PRNA) e o dia da ocorrência do ictus. Utilizou-se como critério de exclusão a presença de doenças neurodegenerativas, sequela de traumatismo crânioencefálico (TCE) ou trauma raquimedular (TRM), doenças neuromusculares, cirurgia prévia de bexiga, pacientes do gênero masculino com hiperplasia prostática benigna (HPB), adenocarcinoma de próstata e prostatectomia prévia, pacientes femininas multíparas com quatro partos ou mais ${ }^{15}$, obstrução infra-vesical (Schafer III ou mais) presente no estudo urodinâmico (EUD), nefropatia, antecedentes de quimioterapia e radioterapia, e pacientes com infecção do trato urinário de repetição e/ou com infecção sintomática no ato da admissão ${ }^{16}$.

Dos 337 prontuários analisados foram excluídos do estudo 205 pacientes. Destes, treze (13) eram crianças/ adolescentes ${ }^{17}$, vinte (20) não haviam realizado exame de imagem da tomografia computadorizada (TC) e ressonância nuclear magnética (RNM) do encéfalo no hospital, quarenta e oito (48) apresentavam alterações urológicas e renais prévias, sete (7) problemas ginecológicos, vinte e um (21) outras doenças cerebrais, sete (7) TCE, oito (8) TRM, sessenta e cinco (65) tinham menos de 6 meses de lesão cerebral, 
nove (9) multíparas ${ }^{15}$, cinco (5) tiveram AVC antes de 2008, e dois (2) pacientes tiveram relato antigo de acidente vascular cerebral isquêmico (AVCi) mas sem repercussões neurológicas sendo admitidos com objetivo de tratamento ortopédico.

Para a coleta das variáveis epidemiológicas, clínicas, radiológicas, laboratoriais e urodinâmicas utilizou-se como critério a primeira consulta que o paciente realizou com a equipe do PRNA, visto que estes pacientes mantêm acompanhamento longitudinal na instituição onde realizou-se a pesquisa e podem apresentar modificações em relação a queixa inicial.

As características clínicas mediante o tipo de AVC, história patológica pregressa, medicações em uso, queixas urinárias, presença de afasia motora ou mista e as alterações cognitivas, como: a memória, capacidade de raciocínio, cálculos, orientação no tempo, espaço e pessoa, compreensão dos comandos verbais e o comportamento foram coletadas na anamnese clínica e exame neurológico. Essa avaliação é realizada pela equipe do programa composta por médicos e enfermeiros, através das informações repassadas pelo paciente, família e cuidadores, levando em consideração o quadro cognitivo e comportamental do paciente. Após está avaliação, quando necessário o mesmo é submetido ao estudo urodinâmico para melhor definição do tipo de bexiga neurogênica e conduta a ser instituída.

Para a coleta de dados em relação ao comportamento miccional antes e após a ocorrência do AVC, os pacientes foram classificados em três grupos: continentes vesicais, com sintomas do trato urinário inferior e incontinência urinária continua. A classificação foi baseada na descrição realizada por Abrams et al. ${ }^{5}$ em queixas de armazenamento e esvaziamento. As queixas de armazenamento compreendem: urgência, urgeincontinência urinária, incontinência urinária de esforço, polaciúria, noctúria e incontinência urinária continua; e as queixas de esvaziamento: hesitação, esforço miccional e disúria.

Para as variáveis radiológicas utilizou-se a classificação de lesão supratentorial e infratentorial. A coleta foi realizada por um único radiologista da instituição na qual foi realizada a pesquisa, através da avaliação direta do exame de imagem da tomografia computadorizada e ressonância nuclear magnética do encéfalo, evitando assim divergências no viés destas informações.

Para a realização deste estudo a amostra necessária seria de 126 pacientes (n), dimensionada através do software G*Power, versão 3.1.7, para responder ao principal objetivo da pesquisa. Para tanto, foram analisados 132 para os quais foram adotados, como parâmetros, o teste qui-quadrado para tabelas de contingencia $2 \times 2$, a probabilidade de significância de 0,05 , poder do teste de 0,80 e o efeito amostral ( $w$, effect size) igual a $0,25^{18}$.

Os dados coletados foram inseridos no programa Sharepoint e após transferidos para um banco de dados no programa Excel $^{R}$ e analisadas no pacote de dados IBM ${ }^{\circledR}$ SPSS $®$ (versão 20). Os resultados foram avaliados por meio de estatística descritiva. As variáveis contínuas foram avaliadas preferencialmente por meio do teste estatístico t-Student, e as categorias, pelos testes não paramétricos (Qui-quadrado e Mann-Whitney), de acordo com a distribuição normal dos dados. A probabilidade de significância considerada na análise estatística foi de 0,05. Todos os testes estatísticos são bilaterais.

\section{RESULTADOS}

Dos 132 pacientes incluídos neste estudo, 80,3\% $(n=106)$ eram portadores de sequela de Acidente Vascular Cerebral Isquêmico (AVCi), e 19,7\% ( $n=26)$ com Acidente Vascular Cerebral Hemorrágico (AVCh). A média de idade foi de $56 \pm 13,1$ anos. O tempo médio da ocorrência do AVC foi de 21,4 meses, sendo calculado entre a data da primeira consulta do paciente com a equipe do Programa de Reabilitação Neurológica (PRNA) e a data da ocorrência do AVC. Em relação as alterações cognitivas e afasia, 31,1\% $(n=41)$ tinham alterações cognitivas em pelo menos um domínio, e 25,8\% ( $n=34)$ apresentavam afasia. Dos pacientes afásicos, 14,4\% ( $n=19)$ apresentavam afasia global, e 11,4\% $(n=15)$ tinham afasia de predomínio motor.

Em relação ao tipo de imagem encefálica, 89,4\% $(n=118)$ realizaram TC, e 10,6\% (n=14) RNM. Em relação ao território vascular, houve predomínio de comprometimento de $66,7 \%$ $(\mathrm{n}=88)$ da artéria cerebral média.

Dos 132 pacientes, 35,6\% $(n=47)$ eram continentes vesicais; $45,5 \%(n=60)$ com sintomas do trato urinário inferior (STUI), e 18,9\% ( $n=25)$ com incontinência urinária continua. A incontinência urinária contínua apresentou relação significativa com a ocorrência de lesões extensas acometendo a área frontal-parietal-temporal-occiptal $(p=0,001)$, alterações cognitivas $(p=0,001)$ e afasia $(p=0,001)$.

Em relação aos sintomas do trato urinário inferior, considerando a classificação das queixas de armazenamento, observamos que a incontinência urinária contínua, polaciúria e urgência foram os sintomas mais evidentes. Quanto às queixas de esvaziamento, a hesitação e o esforço miccional apresentaram maior percentual. Ao analisarmos o comportamento miccional dos pacientes em relação ao tipo de AVC não evidenciamos diferenças estatísticas entre os grupos isquêmico e hemorrágico (Tabela 1). 
Tabela 1. Número de pacientes segundo o comportamento miccional em relação ao tipo de AVC ( $n=132)$.

\begin{tabular}{|c|c|c|c|c|}
\hline Comportamento miccional $^{(*)}$ & $\begin{array}{c}\text { População total }(n=132) \\
\%(n)\end{array}$ & $\begin{array}{c}\text { AVCi }(n=106) \\
\%(n)\end{array}$ & $\begin{array}{c}\operatorname{AVCh}(n=26) \\
\%(n)\end{array}$ & Valor de $p$ \\
\hline Continência urinária & $35,6(47)$ & $38,7(41)$ & $23,0(06)$ & 0,207 \\
\hline \multicolumn{5}{|l|}{ Armazenamento } \\
\hline Incontinência urinária contínua & $18,9(25)$ & $18,8(20)$ & $19,2(05)$ & 0,999 \\
\hline Polaciúria & $18,2(24)$ & $17,0(18)$ & $23,0(06)$ & 0,570 \\
\hline Urgência & $15,2(20)$ & $14,1(15)$ & $19,2(05)$ & 0,545 \\
\hline Urgeincontinência urinária & $13,6(18)$ & $13,2(14)$ & $15,4(04)$ & 0,755 \\
\hline Noctúria & $9,1(12)$ & $9,4(10)$ & $7,7(02)$ & 0,999 \\
\hline \multicolumn{5}{|l|}{ Esvaziamento } \\
\hline Hesitação & $22,7(30)$ & $21,7(23)$ & $27,0(07)$ & $0,758^{(* *)}$ \\
\hline Esforço miccional & $10,6(14)$ & $11,3(12)$ & $7,7(02)$ & 0,736 \\
\hline Disúria & $6,1(08)$ & $5,7(06)$ & $7,7(02)$ & 0,656 \\
\hline
\end{tabular}

* O mesmo paciente poderá apresentar mais de um sintoma nas queixas de armazenamento e esvaziamento, ou em ambas; com exceção da incontinência urinária continua. Valor de $p$ : refere-se ao cálculo estatístico entre o grupo de AVCi e AVCh. Teste Exato de Fisher. **Teste Qui-quadrado com correção de Yates.

Os resultados das Tabelas 2 e 3, analisou 129 pacientes para identificar se a lesão cerebral influenciou ou não o paciente a apresentar disfunção vesical. Três foram excluídos, devido à impossibilidade da correta identificação da área de lesão. O lobo insula $(n=6)$ não apresentou diferença estatística em relação ao comportamento vesical. Quando analisados os resultados entre o lobo cerebral afetado e os sintomas urinários, observamos que lesões pequenas acometendo um único lobo não tiveram influência no comportamento urinário (Tabela 2).

Tabela 2. Correlação do lobo lesionado com os sintomas do trato urinário inferior ( $n=129)$.

\begin{tabular}{|c|c|c|c|c|c|c|c|c|c|}
\hline \multirow{2}{*}{$\begin{array}{c}\text { Lobo } \\
\text { lesionado }\end{array}$} & \multicolumn{9}{|c|}{$\begin{array}{c}\text { Sintomas do trato urinário inferior } \\
\text { n (\%) } \\
\text { valor de } p\end{array}$} \\
\hline & $\begin{array}{l}\text { Urgência } \\
(n=20)\end{array}$ & $\begin{array}{c}\text { UIU } \\
(n=18)\end{array}$ & $\begin{array}{c}\text { IUE } \\
(n=03)\end{array}$ & $\begin{array}{l}\text { Polaciúria } \\
(n=24)\end{array}$ & $\begin{array}{c}\text { Noctúria } \\
(n=12)\end{array}$ & $\begin{array}{l}\text { Hesitação } \\
(n=30)\end{array}$ & $\begin{array}{c}\text { EM } \\
(n=14)\end{array}$ & $\begin{array}{l}\text { Disúria } \\
(n=08)\end{array}$ & $\begin{array}{c}\text { IUC } \\
(n=23)\end{array}$ \\
\hline $\begin{array}{l}\text { Frontal } \\
(n=08)\end{array}$ & $\begin{array}{c}01(5,0) \\
0,999\end{array}$ & $\begin{array}{c}01(5,5) \\
0,999\end{array}$ & $\begin{array}{l}0(0,0) \\
0,999\end{array}$ & $\begin{array}{c}02(8,3) \\
0,642\end{array}$ & $\begin{array}{l}0(0,0) \\
0,999\end{array}$ & $\begin{array}{c}02(6,7) \\
0,999\end{array}$ & $\begin{array}{c}01(7,1) \\
0,999\end{array}$ & $\begin{array}{c}01(12,5) \\
0,410\end{array}$ & $\begin{array}{c}01(4,0) \\
0,999\end{array}$ \\
\hline $\begin{array}{l}\text { Parietal } \\
(n=20)\end{array}$ & $\begin{array}{c}04(20,0) \\
0,513\end{array}$ & $\begin{array}{c}05(27,8) \\
0,156\end{array}$ & $\begin{array}{l}0(0,0) \\
0,999\end{array}$ & $\begin{array}{c}05(20,8) \\
0,531\end{array}$ & $\begin{array}{c}01(8,3) \\
0,690\end{array}$ & $\begin{array}{c}03(10,0) \\
0,405\end{array}$ & $\begin{array}{c}01(7,1) \\
0,695\end{array}$ & $\begin{array}{l}0(0,0) \\
0,357\end{array}$ & $\begin{array}{l}2(8,0) \\
0,525\end{array}$ \\
\hline $\begin{array}{c}\text { Temporal } \\
(\mathrm{n}=02)\end{array}$ & $\begin{array}{l}0(0,0) \\
0,999\end{array}$ & $\begin{array}{c}01(5,5) \\
0,261\end{array}$ & $\begin{array}{l}0(0,0) \\
0,999\end{array}$ & $\begin{array}{l}0(0,0) \\
0,999\end{array}$ & $\begin{array}{l}0(0,0) \\
0,999\end{array}$ & $\begin{array}{l}0(0,0) \\
0,999\end{array}$ & $\begin{array}{l}0(0,0) \\
0,999\end{array}$ & $\begin{array}{l}0(0,0) \\
0,999\end{array}$ & $\begin{array}{l}0(0,0) \\
0,999\end{array}$ \\
\hline $\begin{array}{c}\text { Occiptial } \\
(\mathrm{n}=01)\end{array}$ & $\begin{array}{c}0(0,0) \\
0,999\end{array}$ & $\begin{array}{c}0(0,0) \\
0,999\end{array}$ & $\begin{array}{c}0(0,0) \\
0,999\end{array}$ & $\begin{array}{c}0(0,0) \\
0,999\end{array}$ & $\begin{array}{c}0(0,0) \\
0,999\end{array}$ & $\begin{array}{c}0(0,0) \\
0,999\end{array}$ & $\begin{array}{l}0(0,0) \\
0,999\end{array}$ & $\begin{array}{c}0(0,0) \\
0,999\end{array}$ & $\begin{array}{l}0(0,0) \\
0,999\end{array}$ \\
\hline
\end{tabular}

Valor de p: Teste Exato de Fisher. UIU: urgeincontinência urinária; IUE: incontinência urinária de esforço; EM: esforço miccional; IUC: incontinência urinária continua.

Tabela 3. Correlação da área de lesão cerebral com o comportamento miccional (n=129).

\begin{tabular}{|c|c|c|c|c|c|c|c|c|c|}
\hline \multirow[t]{2}{*}{ Área de lesão } & \multicolumn{9}{|c|}{$\begin{array}{l}\text { Sintomas do trato urinário inferior } \\
\text { n (\%) } \\
\text { valor-p }\end{array}$} \\
\hline & $\begin{array}{c}\text { Urgência } \\
(\mathbf{n = 2 0 )}\end{array}$ & $\begin{array}{c}\text { UIU } \\
(n=18)\end{array}$ & $\begin{array}{c}\text { IUE } \\
(n=03)\end{array}$ & $\begin{array}{c}\text { Polaciúria } \\
(n=24)\end{array}$ & $\begin{array}{c}\text { Noctúria } \\
(n=12)\end{array}$ & $\begin{array}{c}\text { Hesitação } \\
(\mathbf{n}=30)\end{array}$ & $\begin{array}{c}\text { EM } \\
(n=14)\end{array}$ & $\begin{array}{l}\text { Disúria } \\
(n=08)\end{array}$ & $\begin{array}{c}\text { IUC } \\
(n=23)\end{array}$ \\
\hline $\begin{array}{c}\text { FP } \\
(n=35)\end{array}$ & $\begin{array}{c}09(45,0) \\
\mathbf{0 , 0 5 0} *\end{array}$ & $\begin{array}{c}09(50,0) \\
\mathbf{0 , 0 4 2} *\end{array}$ & $\begin{array}{c}03(100,0) \\
\mathbf{0 , 0 1 9}\end{array}$ & $\begin{array}{c}11(45,8) \\
\mathbf{0 , 0 4 2} *\end{array}$ & $\begin{array}{c}03(25,0) \\
0,999\end{array}$ & $\begin{array}{c}10(33,3) \\
0,524^{*}\end{array}$ & $\begin{array}{c}04(28,6) \\
0,999\end{array}$ & $\begin{array}{c}03(37,5) \\
0,682\end{array}$ & $\begin{array}{c}02(8,7) \\
0,053^{*}\end{array}$ \\
\hline $\begin{array}{c}\text { FPT } \\
(n=23)\end{array}$ & $\begin{array}{l}0(0,0) \\
0,023\end{array}$ & $\begin{array}{l}0(0,0) \\
0,042\end{array}$ & $\begin{array}{l}0(0,0) \\
0,999\end{array}$ & $\begin{array}{c}01(4,2) \\
0,074\end{array}$ & $\begin{array}{c}02(16,7) \\
0,999\end{array}$ & $\begin{array}{c}05(16,7) \\
0,999^{*}\end{array}$ & $\begin{array}{l}0(0,0) \\
0,073\end{array}$ & $\begin{array}{l}0(0,0) \\
0,349\end{array}$ & $\begin{array}{c}07(30,4) \\
0,128\end{array}$ \\
\hline $\begin{array}{c}\text { FPO } \\
(n=04)\end{array}$ & $\begin{array}{l}0(0,0) \\
0,999\end{array}$ & $\begin{array}{c}01(22,2) \\
0,999\end{array}$ & $\begin{array}{l}0(0,0) \\
0,999\end{array}$ & $\begin{array}{c}01(16,7) \\
0,999\end{array}$ & $\begin{array}{l}0(0,0) \\
0,999\end{array}$ & $\begin{array}{c}02(13,3) \\
0,999\end{array}$ & $\begin{array}{c}02(28,5) \\
0,999\end{array}$ & $\begin{array}{l}0(0,0) \\
0,999\end{array}$ & $\begin{array}{l}0(0,0) \\
0,999\end{array}$ \\
\hline $\begin{array}{c}\text { PT } \\
(n=01)\end{array}$ & $\begin{array}{c}01(1,0) \\
0,999\end{array}$ & $\begin{array}{l}0(0,0) \\
0,999\end{array}$ & $\begin{array}{l}0(0,0) \\
0,999\end{array}$ & $\begin{array}{l}0(0,0) \\
0,999\end{array}$ & $\begin{array}{l}0(0,0) \\
0,999\end{array}$ & $\begin{array}{l}0(0,0) \\
0,999\end{array}$ & $\begin{array}{c}01(1,0) \\
0,999\end{array}$ & $\begin{array}{c}01(1,0) \\
0,999\end{array}$ & $\begin{array}{l}0(0,0) \\
0,999\end{array}$ \\
\hline $\begin{array}{c}\text { PTO } \\
(n=04)\end{array}$ & $\begin{array}{l}0(0,0) \\
0,999\end{array}$ & $\begin{array}{l}0(0,0) \\
0,999\end{array}$ & $\begin{array}{l}0(0,0) \\
0,999\end{array}$ & $\begin{array}{l}0(0,0) \\
0,999\end{array}$ & $\begin{array}{c}01(33,3) \\
0,327\end{array}$ & $\begin{array}{l}0(0,0) \\
0,999\end{array}$ & $\begin{array}{l}0(0,0) \\
0,999\end{array}$ & $\begin{array}{c}01(50,0) \\
0,228\end{array}$ & $\begin{array}{l}0(0,0) \\
0,999\end{array}$ \\
\hline $\begin{array}{c}\text { TO } \\
(n=01)\end{array}$ & $\begin{array}{l}0(0,0) \\
0,999\end{array}$ & $\begin{array}{c}0(0,0) \\
0,999\end{array}$ & $\begin{array}{c}0(0,0) \\
0,999\end{array}$ & $\begin{array}{c}0(0,0) \\
0,999\end{array}$ & $\begin{array}{c}01(10,0) \\
0,090\end{array}$ & $\begin{array}{c}01(3,0) \\
0,230\end{array}$ & $\begin{array}{c}01(8,0) \\
0,111\end{array}$ & $\begin{array}{c}01(1,0) \\
0,999\end{array}$ & $\begin{array}{l}0(0,0) \\
0,999\end{array}$ \\
\hline $\begin{array}{c}\mathrm{FaO} \\
(n=16)\end{array}$ & $\begin{array}{c}03(15,0) \\
0,714\end{array}$ & $\begin{array}{c}0(0,0) \\
0,125\end{array}$ & $\begin{array}{c}0(0,0) \\
0,999\end{array}$ & $\begin{array}{c}02(8,3) \\
0,735\end{array}$ & $\begin{array}{c}02(16,7) \\
0,644\end{array}$ & $\begin{array}{c}04(13,3) \\
0,999\end{array}$ & $\begin{array}{c}01(7,1) \\
0,999\end{array}$ & $\begin{array}{c}01(12,5) \\
0,999\end{array}$ & $\begin{array}{c}07(30,4) \\
\mathbf{0 , 0 0 1}\end{array}$ \\
\hline
\end{tabular}

Valor de $p$ : Teste Exato de Fisher. * Teste Qui-quadrado com correção de Yates.

UIU: urgeincontinência urinária; IUE: incontinência urinária de esforço; EM: esforço miccional; IUC: incontinência urinária continua; FP: Fronto-parietal; FPT: Fronto-parieto-temporal; FPO: Fronto-parieto-occiptal; PT: Parieto-temporal; PTO: Parieto-temporal-occiptal; TO: temporal-occiptal; FaO: Frontal,parietal,temporal e occiptal. 
Observamos em nossa amostra, que lesões acometendo mais de um lobo são mais suscetíveis de alterar o comportamento miccional (Tabela 3). Houve significância estatística para a presença da lesão em região fronto-parietal e o sintoma de urgência $(p=0,050)$, urgeincontinência urinária $(p=0,042)$, incontinência urinária de esforço $(p=0,019)$ e polaciúria $(p=0,042)$. Comparando a área frontal à occipital houve maior probabilidade de incontinência urinária contínua $(p=0,001)$. Os resultados obtidos na análise da área fronto-parieto-temporal obtivemos significância estatística para a urgência $(p=0,023)$ e urgeincontinência urinária $(p=0,042)$, no entanto, esse resultado ocorreu pela ausência de pacientes com essas queixas para a respectiva área de lesão cerebral.

\section{DISCUSSÃO}

O principal objetivo deste trabalho foi relacionar a topografia da lesão cerebral ocasionada por AVC e o comportamento vesical, visando contribuir com as pesquisas já realizadas acerca do complexo sistema que regula o controle miccional. Nessa amostra, os pacientes analisados encontravam-se na fase crônica do AVC, com leves ou nenhuma modificação nos déficits neurológicos e nas queixas urinárias, sendo uma característica importante a ser observada.

Neste estudo, não houve diferenças entre o tipo de AVC e a frequência dos sintomas entre os grupos, isquêmico e hemorrágico. Achados semelhantes foram encontrados por Gupta et al. ${ }^{19}$, diferindo apenas no tempo de lesão cerebral dos pacientes submetidos ao estudo, onde o intervalo foi de 8-53 dias $^{19}$.

Pacientes na fase aguda apresentam com maior frequência incontinência urinária ou retenção, as quais estão relacionadas à gravidade do edema cerebral, déficits motores, cognitivos, de linguagem e comportamentais que influenciam na capacidade do indivíduo em comunicar-se expressando assim suas necessidades de vida diária ${ }^{20}$. A incontinência urinária pode melhorar durante o primeiro ano da lesão, mas um grande número de pacientes permanece incontinente após este período. Ambos estudos demonstram resultados semelhantes aos descritos em nossa pesquisa, no entanto, não definem qual o tipo de incontinência urinária estava presente nos pacientes pesquisados ${ }^{20,21}$. Pizzi et al. ${ }^{22}$ avaliou 106 pacientes na fase aguda do AVC isquêmico com reavaliação após 30 dias, observando que $79 \%$ eram incontinentes vesicais, a qual foi significativamente associada à idade e incapacidade funcional.

Nas alterações de armazenamento vesical, os sintomas mais frequentes foram polaciúria, urgência e urgeincontinência urinária. Estes sintomas podem ser indicativos de bexiga hiperativa ${ }^{23}$, que é o achado mais comum no estudo urodinâmico em pacientes com $\mathrm{AVC}^{24}$. Em relação às queixas de esvaziamento, as mais prevalentes foram hesitação e esforço miccional. Em estudos prévios, alterações de armazenamento são descritas em 73,3\% no AVCh e 63,6\% nos isquêmicos. Em relação as queixas de esvaziamento, com predominância para o grupo de AVCi com 51,5\% e 13,3\% no hemorrágico ${ }^{25}$. Para Fowler ${ }^{6}$, noctúria esteve presente em $36 \%$ dos pacientes até três meses após o AVC, seguido de $29 \%$ com urgeincontinência, 25\% hesitação, e 6\% retenção urinária. A urgência e urgeincontinência urinária estão associadas à presença de detrusor hiperativo ao longo de semanas ou meses após o $\mathrm{AVC}^{7}$.

Procuramos correlacionar se determinada área de lesão cerebral influenciaria a presença de disfunção vesical. Observamos que na ocorrência de lesões extensas o comportamento miccional foi alterado, ocasionando diferentes tipos de sintomas e em alguns casos a incontinência urinária contínua. Resultados semelhantes foram encontrados por outros autores que relacionam a presença de afasia e alterações cognitivas à incontinência urinária ${ }^{21,26}$.

Tais resultados são condizentes quando correlacionamos com a literatura em relação à fisiopatologia neuro-miccional, em que lesões em lobo frontal desencadeiam urgência, polaciúria e urgeincontinência ${ }^{27}$, também demonstrados em estudos realizados através de SPECT, ao avaliar indivíduos com distúrbios cognitivos, nos quais também houve uma diminuição significativa na atividade do córtex frontal direito relacionado à disfunção urinária ${ }^{28}$. No lobo parietal encontra-se o córtex somatossensorial primário, que representa, dentre várias partes do corpo, a pelve e genitálias e, lesões nessa área resultam em alterações na percepção sensorial. Ambos os lobos frontal e parietal estão relacionados ao processo de decisão, percepção miccional e avaliação social para o esvaziamento vesical ${ }^{29}$. O controle voluntário da micção é um complexo sistema, no qual lesões em lobo fronto-parietal e regiões subcorticais adjacentes são susceptíveis de serem afetadas no AVC, contribuindo para alterações no sistema de controle voluntário da micção $0^{30}$.

A partir do lobo frontal as fibras neuronais projetarse-iam sobre os núcleos subcorticais incluindo o tálamo, sistema límbico e gânglios da base. No tálamo, as fibras provenientes da medula estimulariam a sensação da bexiga cheia, despertando o cérebro para a necessidade de urinar $^{31}$. Nesse momento, o indivíduo tem a capacidade de avaliar se o ambiente e o momento estão adequados para iniciar a micção, pela capacidade de julgamento, atenção e percepção. O lobo frontal está associado à incontinência urinária, urgência, frequência e urgeincontinência ${ }^{6}$. 
Os lobos frontal e parietal exercem atividades no controle miccional, como já descrito por alguns autores,6,27,29-31. Estudos com imagem de ressonância magnética funcional (IRMf) têm sido usados na tentativa de identificar as áreas cerebrais envolvidas no controle voluntário da micção, e a ativação dos gânglios basais, córtex parietal, sistema límbico e cerebelo podem apoiar a hipótese de que um complexo sistema sensório motor está envolvido no controle inibitório do reflexo miccional ${ }^{32}$.

Em lesões extensas acometendo área frontal a occipital, houve significância estatística para a presença de incontinência urinária contínua. Múltiplas áreas de lesão são relacionadas com déficits motores importantes, alterações cognitivas, comportamentais e de linguagem, assim como na influência direta do comportamento miccional desses pacientes $^{26}$. Ocorre aumento significativo da atividade no giro frontal superior direito e medial esquerdo, giro parietal e giro do cíngulo na presença de disfunção urinária grave, quando comparado pacientes com sequelas neurológicas importantes com aqueles que apresentam sintomas leves ${ }^{6}$.

Concluímos que a disfunção vesical é frequente em pacientes com AVC. Os resultados demonstram que existe associação entre lesões em área fronto-parietal com disfunção vesical para os sintomas de urgência, urgeincontinência urinária, incontinência urinária de esforço e polaciúria. $\mathrm{Na}$ ocorrência de lesão extensas associado a presença de alterações cognitivas e afasias, observamos que há maior probabilidade de incontinência urinária contínua. Não encontramos correlação estatística entre o tipo de AVC e as queixas de armazenamento e esvaziamento vesical. Entretanto, ainda são necessários mais estudos para este tema, principalmente na caracterização das áreas supra e infratentorial envolvidas, assim como o tamanho da lesão, correlacionando com o tipo de queixa de armazenamento e esvaziamento vesical apresentado pelo paciente.

\section{AGRADECIMENTOS}

À diretoria e aos funcionários da Rede SARAH de Hospitais de Reabilitação.

Ao Dr. Antônio Lopes Jr. (médico radiologista da Rede Sarah de Hospitais de Reabilitação).

\section{REFERÊNCIAS}

1. Sacco RL, Kasner SE, Broderick JP, Caplan LR, Connors JJ, Culebras A, Elkind MS, George MG, Hamdan AD, Higashida RT, Hoh BL, Janis LS, Kase CS, Kleindorfer DO, Lee JM, Moseley ME, Peterson ED, Turan TN, Valderrama AL, Vinters HV; American Heart Association Stroke Council, Council on Cardiovascular Surgery and Anesthesia; Council on Cardiovascular Radiology and Intervention; Council on Cardiovascular and Stroke Nursing; Council on Epidemiology and Prevention; Council on Peripheral Vascular Disease; Council on Nutrition, Physical Activity and Metabolism. An updated definition of stroke for the 21st century: a statement for healthcare professionals from the American Heart Association/American Stroke Association. Stroke 2013;44(7):2064-89. https://doi.org/10.1161/ STR.0b013e318296aeca

2. Natsume O. Detrusor contractility and overactive bladder in patients with cerebrovascular accident. Int J Urol. 2008;15(6):505-10. https://doi.org/10.1111/j.1442-2042.2008.02045.x

3. Han KS, Heo SH, Lee SJ, Jeon SH, Yoo KH. Comparison of urodynamics between ischemic and hemorrhagic stroke patients: can we suggest the category of urinary dysfunction in patients with cerebrovascular accident according to type of stroke? Neurourol Urodyn. 2010;29(3):387-90. https://doi.org/10.1002/nau.20708

4. Brittain KR, Perry SI, Peet SM, Shaw C, Dallosso H, Assassa RP, Williams K, Jagger C, Potter JF, Castleden CM. Prevalence and impact of urinary symptoms among community-dwelling stroke survivors. Stroke. 2000;31(4):886-91. https://doi.org/10.1161/01. STR.31.4.886

5. Abrams P, Cardozo L, Fall M, Griffiths D, Rosier P, Ulmsten U, van Kerrebroeck P, Victor A, Wein A; Standardisation Sub-committee of the International Continence Society. The standardisation of terminology of lower urinary tract function. Neurourol Urodyn. 2002;21(2):167-78. https://doi.org/10.1002/nau.10052

6. Fowler CJ. Neurological disorders of micturition and their treatment. Brain. 1999;122(Pt 7):1213-31. https://doi.org/10.1093/ brain/122.7.1213

7. Ji Li W, Oh SJ. Management of lower urinary tract dysfunction in patients with neurological disorders. Korean J Urol. 2012 Sep;53(9):583-92. https://doi.org/10.4111/kju.2012.53.9.583

8. Olsen-Vetland P. Urinary continence after a cerebrovascular accident. Nurs Stand. 2003;17(39):37-41. https://doi.org/10.7748/ ns.17.39.37.s46

9. Pilcher M, MacArthur J. Patient experiences of bladder problems following stroke. Nurs Stand. 2012;26(36):39-46. https://doi. org/10.7748/ns2012.05.26.36.39.c9087

10. Marques LP, Schneider IJC, Giehl MWC, Antes DL, d'Orsil E. Fatores demográficos, condições de saúde e hábitos de vida associados à incontinência urinária em idosos de Florianópolis, Santa Catarina. Rev Bras Epidemiol. 2015;18(3):595-606. https:// doi.org/10.1590/1980-5497201500030006

11. Pitangui ACR, Silva RG da, Araújo RC de. Prevalência e impacto da incontinência urinária na qualidade de vida de idosas institucionalizadas. Rev Bras Geriatr Gerontol. 2012;15:619-26. https://doi.org/10.1590/\$1809-98232012000400002

12. Neves RCS. Incidência e fatores de risco de bexiga hiperativa em adultos: resultados de um estudo prospectivo de base populacional [Internet]. 2010 [capturado 2017 Jul 4] Disponível em: http://www. arca.fiocruz.br/handle/icict/4312

13. Datasus. Classificação estatística internacional de doenças e problemas relacionados à saúde [Internet]. 2008. Disponível em: http://www.datasus.gov.br/cid10/V2008/cid10.htm

14. Green JB. Brain reorganization after stroke. Top Stroke Rehabil. 2003; 10(3):1-20. https://doi.org/10.1310/H65X-23HW-QL1G-KTNQ

15. Scarpa KP, Herrmann V, Palma PCR, Riccetto CLZ, Morais SS. Sintomas urinários irritativos após o parto vaginal ou cesárea. Rev Assoc Med Bras. 2009;55(4):416-20. https://doi.org/10.1590/ S0104-42302009000400016 
16. Tanagho EA, Mcaninch JW. Urologia geral de Smith. $16^{\circ}$ ed. Barueri: Manole; 2007, 860 p.

17. Estatuto da criança e do adolescente. Lei no 8.069, 13 de julho de 1990, e legislação correlate [Internet]. 9a ed. Biblioteca Digital da Câmara dos Deputados. Central de Documentação e Informação. Coordenação de Biblioteca. 2012. Disponível em: http://bd.camara. gov.br

18. Cohen JA. A power primer. Psychol Bull. 1992;112(1):155-9. https:// doi.org/10.1037/0033-2909.112.1.155

19. Gupta A, Taly AB, Srivastava A, Thyloth M. Urodynamics post stroke in patients with urinary incontinence: Is there correlation between bladder type and site of lesion? Ann Indian Acad Neurol. 2009 Apr;12(2):104-7. https://doi.org/10.4103/0972-2327.53078

20. Gelber D, Good D, Laven L, Verhulst S. Causes of urinary incontinence after acute hemispheric stroke. Stroke. 1993;24(3):378-82. https:// doi.org/10.1161/01.STR.24.3.378

21. McKenzie P, Gopal BH. The incidence and etiology of overactive bladder in patients after cerebrovascular accident. Curr Urol Rep. 2012;13(5):402-6. https://doi.org/10.1007/s11934-012-0269-6

22. Pizzi A, Falsini C, Martini M, Rossetti A M, Verdesca S, Tosto A. Urinary incontinence after ischemic stroke: clinical and urodynamic studies. Neurourol Urodyn. 2014 Apr;33(4):420-5. https://doi. org/10.1002/nau.22420

23. Sogari $P$, Dambroa $M$, Souto $C A$. Avaliação urodinâmica do homem com obstrução não neurogênica do trato urinário inferior. Rev AMRIGS. 2000;44(34):159-63.

24. Chen YC, Liao YM, Kuo HC. Lower urinary tract dysfunction in stroke patients. JUTA. 2007;18:147-50

25. Ersoz M, Tunc H, Ozel SM. Bladder storage and emptying disorder frequencies in hemorrhagic and ischemic stroke patients with bladder dysfunction. Cerebrovasc Dis. 2005;20(5):395-9. https:// doi.org/10.1159/000088670

26. Meng NH, Lo SF, Chou LW, Yang PY, Chang CH, Chou ECL. Incomplete bladder emptying in patients with stroke: is detrusor external sphincter dyssynergia a potential cause? Arch Phys Med Rehabil. 2010 Jul;91(7):1105-9. https://doi.org/10.1016/ j.apmr.2010.03.017

27. SBU: Sociedade Brasileira de Urologia. Disfunções miccionais em doenças neurológicas:infecciosas-inflamatórias-degenerativas. Brasília: AMB-CFM; 2006.

28. Sakakibara R, Uchida Y, Ishii K, Kazui H, Hashimoto M, Ishikawa M, Yuasa $T$, Kishi M, Ogawa E, Tateno F, Uchiyama T, Yamamoto T, Yamanishi T, Terada H; SINPHONI (Study of Idiopathic Normal Pressure Hydrocephalus On Neurological Improvement). Correlation of right frontal hypoperfusion and urinary dysfunction in iNPH: a SPECT study. Neurourol Urodyn. 2012;31(1):50-5. https:// doi.org/10.1002/nau.21222

29. Haines DE. Neurociência fundamental: com aplicações básicas e clínicas. 3a ed. Rio de Janeiro: Elsevier; 2006, 653 p.

30. Pettersen R, Haig Y, Nakstad PH, Wyller TB. Subtypes of urinary incontinence after stroke: relation to size and location of cerebrovascular damage. Age Ageing. 2008;37(3):324-7. https:// doi.org/10.1093/ageing/afm196

31. Barata HS, Carvalhal GF. Urologia: princípios e praticas. Porto Alegre: Artmed; 1999.

32. Zhang H, Reitz A, Kollias S, Summers P, Curt A, Schurch B. An fMRI study of the role of suprapontine brain structures in the voluntary voiding control induced by pelvic floor contraction. Neuroimage. 2005;24(1):174-80. https://doi.org/10.1016/j. neuroimage.2004.08.027 